\title{
El uso de la biblioteca y de la tecnología de la información y comunicación para la investigación entre los estudiantes universitarios de Río de Janeiro: diferencias de género y socioculturales
}

\author{
Cládice Nóbile Diniz \\ UniABEU / UniverCidade (Brasil)
}

Gilda Olinto de Oliveira

Instituto Brasileiro de Informação em Ciência e Tecnologia (IBICT) (Brasil)

\section{Resumen}

El presente trabajo analiza el uso de la biblioteca y de la tecnología de información y comunicación (TIC) en relación con la búsqueda de datos para la investigación entre estudiantes universitarios de Río de Janeiro. Los aspectos del uso de la biblioteca considerados incluyen la frecuencia y la finalidad del uso, así como la evaluación por parte de los alumnos de su propio éxito en la localización de la información. También se analizan las habilidades básicas en TIC, consideradas necesarias en este nivel de enseñanza, de acuerdo con lo que establece la literatura internacional. Estas incluyen, entre otras cosas, el uso de Internet en las tareas de investigación, y en concreto la búsqueda de información mediante buscadores. Se intenta también identificar las características del ambiente sociocultural del alumno y las que pueden ser determinantes en las diferencias encontradas en cuanto a las habilidades de uso de la biblioteca y de las TIC. Entre los aspectos socioculturales considerados en el estudio por su posible influencia en la adquisición de habilidades en TIC destacan el sexo de los alumnos, su origen social y las inversiones realizadas en actividades culturales. Entre las inversiones específicas y experiencias que pueden favorecer la fluidez en tecnologías de información y comunicación se ha considerado la presencia de un ordenador en el medio doméstico, la edad en que el alumno se inicia en las TIC, la fluidez en lengua inglesa y la asistencia a cursos extraescolares destinados a aprender dichas tecnologías. La inclusión del factor sexo en el presente estudio se debe a que diversos estudios demuestran que la participación de las mujeres en algunas áreas de las ciencias y de la tecnología es todavía escasa. De mantenerse esta situación, la inserción de las mujeres en la sociedad de la información se vería perjudicada a largo plazo. El

Scire. $13: 2$ (jul.-dic. 2007) 139-157. ISSN 1135-3716. 
estudio se llevó a cabo durante 2004 y se centró en los estudiantes de tercer curso de Administración de dos grandes universidades privadas de la ciudad de Río de Janeiro. Respondieron al cuestionario 320 alumnos, aproximadamente el 77\% del universo de la investigación.

Palabras clave: Fluidez en las tecnologías de información y de comunicación (TIC). Alfabetización en información. Recursos humanos en tecnologías de información y comunicación. Enseñanza de Administración.

\section{Abstract}

This paper focuses on aspects of library use, as well as on information and communication technologies (ICT) use in the process of information search for research purposes among college students. Dimensions of library use that were measured include frequency and objective of use, and evaluation of success of use by the students. With respect to the use of information and communication technologies, an effort was made to deal with basic dimensions of ICT fluency as considered by international literature on the subject. These include, among others, the frequency of internet use and the acquaintance of students with search engines. Another emphasis of this paper is to try to identify how the socio-cultural environment of the student can determine his TIC fluency. Among these sociocultural determinants of ICT fluency that were dealt with here are the student gender, the family social origins and the investments made in the acquisition of cultural and technical resources. Cultural and technical resources that are taken into consideration as possible influences in the acquisition of fluency in ICT are the presence of computer at home and early access to it, as well as fluency in English and attendance to specific extra-curricular courses in ICT. The inclusion of gender factor in the study of ICT fluency results from several evidences showing that women's participation in certain areas of science and technology is still restrict. The maintenance of this situation, in the long run, will impair women's participation in information society. The empirical study was undertaken during the year of 2004 and applied to the population of students of the third school year of Business Schools of two large Rio de Janeiro private universities; 320 students answered to the questionnaire, which had a response rate of about $77 \%$ of the target population.

Key words: Fluency with information and communication technologies (ICT). Information literacy. Human resources in information technology (IT). Business School evaluation.

\section{Introducción}

El presente trabajo se basa en los datos obtenidos en el estudio realizado entre estudiantes universitarios para identificar el acceso a las tecnologías de información y comunicación (TIC), así como sus habilidades en el uso de las mismas, la 
utilización que hacen de las TIC para sus tareas de investigación y sus opiniones en cuanto a la necesidad y las perspectivas de aplicación de las TIC en sus futuras actividades profesionales (Diniz, 2005).

Se intenta también definir el uso y la visión del estudiante acerca del papel de la biblioteca universitaria en el contexto actual, en el cual se observa una valorización y utilización crecientes de las tecnologías de información para las tareas de investigación.

Además de procurar brindar un marco de contacto de los estudiantes universitarios con las TIC, este estudio busca también indicios de aspectos socioculturales que puedan ser relevantes para garantizar la capacitación en estas tecnologías. En este sentido, destaca especialmente el sexo del alumno, su origen social del alumno y algunas actividades extraescolares y hábitos que pueden favorecer dicha capacitación.

Los datos descritos en este estudio se refieren a alumnos que están en la última fase del curso de graduación y, por lo tanto, prácticamente listos para integrarse en el mercado de trabajo como profesionales. El área académica seleccionada para el estudio fue la carrera de Administración y se incluyeron unidades de enseñanza situadas en diferentes regiones de la ciudad de Río de Janeiro.

\section{Las TIC en la formación del estudiante y en la preparación para la actividad de investigación}

Es conocida la importancia que tiene la capacitación del estudiante universitario en el uso de las TIC, pues estas tecnologías, además de abarcar actualmente todas las esferas de la vida social, contribuyen a su inserción en un mercado de trabajo propio de una economía cada vez más globalizada. Las TIC constituyen actualmente un aspecto destacado de la calificación laboral, específicamente en el mundo empresarial, tal como se ha comprobado en diversos estudios sobre el tema (Daniel y Cox, s. f.).

La relevancia del dominio de las TIC por parte de los estudiantes no se restringe solo a la garantía de sus perspectivas individuales, sino también a las oportunidades de los países en vías de desarrollo de integrarse en la nueva economía, ya que las pruebas demuestran que el avance económico de los países más desarrollados se apoya desde hace mucho tiempo en las TIC (Mann, 2004; OCDE, 2002).

No obstante, a pesar de que las tecnologías de información y comunicación son una parte esencial de las perspectivas de desarrollo en el mundo actual, la introducción y la difusión de las mismas no garantizan la promoción social. Se ha atribuido a las TIC la capacidad de convivir con diversos tipos de desequilibrios sociales, sugiriendo que incluso pueden estar favoreciendo el aumento de ciertas desigualdades: las que existen entre países desarrollados y países en vías de

Scire. 13 : 2 (jul.-dic. 2007) 139-157. ISSN 1135-3716. 
desarrollo, y las que se dan entre regiones y entre grupos sociales. Este tipo de constatación hace cada vez más urgente la actuación política al respecto y el intercambio de experiencias entre países que necesitan dar un salto digital para evitar la marginalización de regiones y de grandes segmentos poblacionales (Castells, 2003; ECLAC, 2003; Chu, 2002; Pohjola, 2002).

Debido a la precaria situación de los países en vías de desarrollo en lo que se refiere al acceso a las nuevas TIC, los estudios y las iniciativas en esos países se concentran en la descripción de la exclusión digital de los estratos pobres, así como en las políticas necesarias para su superación. No obstante, sobre todo en países desarrollados, se han llevado a cabo varios esfuerzos para garantizar la adquisición de competencias en TIC entre los alumnos de enseñanza superior, teniendo como objetivo su preparación para el mercado de trabajo, identificando los aspectos fundamentales de la fluidez en dichas tecnologías, desarrollando mecanismos para medirla y formulando políticas y acciones para promoverla.

La caracterización de la fluidez en tecnología de información y comunicación fue desarrollada por la comisión de alfabetización digital de la Academia Norteamericana de Ciencias (National Academy of Science), según describe el Committee (1999), basándose en la necesidad de transmitir información y comportamientos adecuados al estudiante universitario para que este no solo adquiera los conocimientos específicos de su titulación, sino que también aprenda a reformularlos y expresarlos a través de los medios tecnológicos actualmente disponibles para los profesionales, así como a influir en el contexto sociocultural en el que actúa o va a actuar, es decir, a valerse de las TIC de un modo socialmente responsable.

Se puede observar que para alcanzar la fluidez en TIC es necesario adquirir capacidades y habilidades complejas. Estas, para el Committee (1999), abarcan tres aspectos: capacidades intelectuales, dominio de conceptos sobre las TIC y habilidades en su utilización. Hay que destacar también que la fluidez en TIC tiene múltiples áreas de aplicación y para cada área se ha de tener en cuenta, necesariamente, un determinado conjunto de factores.

La citada Academia Norteamericana, desde la publicación en 1999 de su informe de investigación sobre el problema considerado, procuró sensibilizar a innumerables instituciones de enseñanza superior sobre la importancia de garantizar la fluidez en tecnologías de información y comunicación a sus estudiantes. A esta iniciativa le siguieron otras de diferentes instituciones, y los investigadores aportaron sus visiones acerca de qué es lo que conforma la fluidez en TIC y qué métodos se pueden utilizar para medirla. A partir de entonces se fueron publicando cada vez más textos que abordan los problemas relacionados con el tema. Se observó también que esos autores parten esencialmente de la caracterización del Comité antes mencionado, ajustándola a un tipo de graduación específica y a los instrumentos de medida, que son, en general, cuestionarios (Diniz, 2005). 
Asimismo destacan entre las instituciones de enseñanza superior de los países desarrollados diversas iniciativas on-line para el desarrollo de la information literacy, las llamadas tutorías, que hacen hincapié sobre todo en la preparación para la investigación independiente y creativa mediante el uso intensivo de herramientas tecnológicas (Hatschbach, 2002). Las iniciativas de este tipo aún no están muy difundidas en los países menos avanzados.

\section{Cultura, origen social, género y fluidez en tecnología de la información y comunicación}

Además de ser compleja y abarcar varios aspectos, la adquisición de competencias en tecnología de información y comunicación parece depender de muchos factores. Para su uso independiente y creativo no parece ser suficiente el solo hecho de tener acceso al ordenador y aprender a usarlo. Se ha dicho, por ejemplo, que el aprendizaje de esas tecnologías para el dominio de la actividad de investigación y para la resolución de problemas depende del conocimiento de la lengua inglesa, ya que prácticamente el $90 \%$ de las páginas de web están en ese idioma (Bonder, 2001). Es importante señalar que el conocimiento del inglés en Brasil, así como en otros países en vías de desarrollo, depende de la oportunidad que el alumno tenga de asistir a clases particulares extraescolares, ya que las escuelas no ofrecen un nivel adecuado y suficiente de enseñanza de esta lengua.

La adquisición de competencias en TIC en Brasil también puede estar vinculada con la asistencia a clases extraescolares de informática, también particulares: son lo que se conoce como cursinhos.

Otra cuestión que se ha de tener en cuenta es la importancia del ambiente doméstico en el aprendizaje, así como el contacto precoz con las TIC por la disponibilidad de ordenador en casa, por la perspectiva de desarrollo de un aprendizaje autónomo y por el acceso a otros tipos de recursos.

También hay que considerar la relevancia del desarrollo de actitudes y valores que pueden ser fundamentales para la adquisición de habilidades en TIC, como los ligados a la autoevaluación, la autoestima y las perspectivas profesionales.

A partir de las características arriba mencionadas se pueden analizar algunos aspectos de los conceptos de capital cultural y capital social difundidos por la teoría de Bourdieu (1979 y 1987). El capital cultural está constituido por disposiciones, preferencias, determinadas elecciones y valores, mientras que el social consiste en relaciones y contactos. Ambos son recursos sutiles, estratégicos y adquiridos o moldeados desde una fase temprana, en el proceso de socialización. Ambos van a contribuir a definir las oportunidades de los individuos, garantizando así ciertas ventajas en el mercado de trabajo. Los miembros de las clases más favorecidas tienden a perpetuar su situación social privilegiada utilizando su capital cultural y

Scire. $13: 2$ (jul.-dic. 2007) 139-157. ISSN 1135-3716. 
social. Por lo tanto, los alumnos cuyos padres tienen una posición social más elevada probablemente tendrán acceso a esos capitales, los cuales, en este contexto, suponen condiciones favorables para la adquisición de TIC. El capital social lo constituyen los contactos y el apoyo en redes informales que contribuyen al incremento del sentimiento interno de poder y autoestima de los individuos, y que podrán favorecer el acceso y la habilidad en las TIC.

Algunos estudios sugieren que las ventajas obtenidas gracias a la condición social y al capital cultural y social a ella asociado se aplican también a la cuestión de las diferencias entre sexos; en este sentido, los hombres cuentan con más facilidades (Martínez, 2005; Olinto, 1994). A pesar de que hombres y mujeres vienen demostrando competencias similares en matemáticas y en las disciplinas ligadas a las ciencias duras, tanto en la enseñanza primaria como en la superior; a pesar de que las mujeres alcanzan niveles educativos incluso más elevados que los hombres, tanto en los países desarrollados como en diversas zonas en vías de desarrollo (con una proporción de ingreso en la universidad igual o mayor que la de los hombres); a pesar de la intensa y progresiva inserción de las mujeres en el mercado laboral; a pesar del aumento de la igualdad entre los géneros en relación con la preparación profesional, las mujeres aún se mantienen en determinados nichos educativos y sus oportunidades profesionales son limitadas y diferentes. Pese a su actividad académica y profesional, las mujeres que se matriculan en la universidad todavía lo hacen en un número muy reducido en determinadas áreas de ciencias y en áreas tecnológicas. Además, tanto en las profesiones llamadas "masculinas" como en las "femeninas", ellas tienen mucha más dificultad para alcanzar altos cargos y posiciones de liderazgo (Mello, Lastres y Marques, 2003; Olinto, 2003 y 2004; García, Escamilla y Reyes, 2004). La persistencia de esas desigualdades, que probablemente se deben al predominio de una cultura de género perjudicial para las mujeres, constituye una amenaza para su inserción en la sociedad de la información en igualdad de condiciones con los hombres. Las consecuencias negativas de esta tendencia afectan a la sociedad como un todo, lo cual ha sido señalado por algunos autores que consideran la búsqueda de mayor equidad entre los géneros, tanto en la educación como en el trabajo, como un hecho fundamental para que países y regiones alcancen un desarrollo socioeconómico rápido y sostenible (Chen, 2004; Sen, 1999).

Entre las actitudes y comportamientos que las mujeres interiorizan como adecuados y que les son asignados en las escuelas y en los centros de trabajo están la elección de carreras consideradas "femeninas" y la percepción de una imagen negativa de sí mismas respecto a su propia habilidad en las áreas de ciencias exactas y tecnología, así como su menor capital social, es decir, su menor capacidad para hacer contactos y buscar información (Martínez, 2005; Quiñones, 2005). También se han observado diferencias en cuanto al aprendizaje de las tecnologías de información y comunicación: a pesar de que hombres y mujeres se desenvuelven de 
un modo similar, la idea de competencia en el terreno informático se asocia normalmente a aquellos. También se considera que están más capacitados para utilizar los recursos de Internet y del software de comunicación y los emplean más adecuada y frecuentemente que las mujeres (Bonder, 2001).

En resumen, se puede decir que los estudios que relacionan factores como la clase social, la cultura y el sexo con el uso diferenciado de las TIC ayudan a determinar el modo en que estos aspectos culturales facilitan o dificultan el acceso a las tecnologías y cómo contribuyen a la perpetuación de las desigualdades sociales. Tales estudios pueden servir de base a políticas dirigidas a superar dichas desigualdades por medio de acciones que tengan como objetivo el desarrollo de los capitales cultural y social entre los sectores socialmente poco privilegiados y entre las mujeres.

\section{Datos y medidas}

Para el estudio se escogió a graduandos brasileños de la carrera de Administración, y entre ellos, a los de universidades privadas. La elección de este tipo de instituciones se debe al papel que tiene el sector privado en la enseñanza superior brasileña hoy en día, ya que organizó el 70,75\% de los cursos de graduación del país en 2002 (Inep/MEC, 2002). Y se optó por la carrera de Administración porque ocupa el primer puesto en número de matrículas y se encuentra en clara expansión: entre 2001 y 2003 prácticamente dobló la cantidad de cursos ofrecidos, que actualmente es de casi 3000 en el territorio nacional.

En cuanto al área geográfica, se optó por recoger datos sobre instituciones de enseñanza superior localizadas en el área metropolitana denominada Grande Rio, la cual abarca la ciudad de Río de Janeiro y la región que la rodea, conocida como Baixada Fluminense. En 2003 esa región contaba con 150 cursos de Administración. De ella se seleccionaron dos grandes centros universitarios privados, uno de los cuales se encuentra entre las diez mayores instituciones privadas de enseñanza en cuanto al número de matrículas.

Ambas instituciones seleccionadas poseen diversas unidades de enseñanza en diferentes regiones del Grande Rio. El número de alumnos que asisten en ellas a los cursos de Administración es de aproximadamente 5000, de los cuales 4000 pertenecen a la más importante de las dos. Se seleccionaron dos unidades de enseñanza de esta última y una de la otra institución.

Mediante la diversificación de las unidades de enseñanza se buscó la inclusión en el estudio de regiones que presentan indicadores socioeconómicos diferenciados, con la finalidad de garantizar un cierto grado de variación de las características socioeconómicas y culturales de los alumnos.

El universo del estudio empírico fueron los estudiantes de tercer curso de Administración de las unidades de enseñanza seleccionadas, lo que supuso la Scire. 13 : 2 (jul.-dic. 2007) 139-157. ISSN 1135-3716. 
obtención de datos sobre cinco grupos diferentes de alumnos. Respondieron al cuestionario elaborado para este estudio un total de 320 estudiantes, es decir, aproximadamente el $70 \%$ de la población abarcada en el estudio.

El cuestionario aplicado a los alumnos pretendía identificar las dimensiones de fluidez en TIC y las de uso de la biblioteca, así como detectar los factores que pueden intervenir en la caracterización de dicha fluidez. Estos factores incluyen el ambiente sociocultural del alumno y algunos aspectos de la experiencia escolar y de las extraescolares, especialmente aquellas relacionadas con el aprendizaje de TIC fuera de la escuela.

Para detectar las habilidades en el uso de las TIC se establecieron dos grupos: en el primero se midieron las habilidades en cuanto al uso del software considerado básico para la formación del alumno en cualquier área de la titulación y en el mercado laboral (Daniel y Cox, s. f.); en el segundo se midieron las habilidades en cuanto al uso de Internet destinado a la búsqueda de información para la investigación en el trabajo académico. Cabe mencionar que este segundo grupo posee indicadores específicos de preparación para la actividad de investigación independiente y creativa.

Además de intentar medir dichas habilidades se incluyeron preguntas destinadas a detectar la valoración y la evaluación del acceso a las tecnologías de información y comunicación por parte del alumno. Hay que destacar que el aspecto de la valoración fue incluido porque actualmente está considerado como un elemento fundamental para la absorción de conocimientos en el proceso de aprendizaje. Por otra parte, la valoración de las TIC indicaría hasta qué punto el alumno es consciente de la importancia que se concede a dichas tecnologías en el mercado de trabajo.

Para medir el uso y el papel de la biblioteca en el contexto actual de la titulación se tuvo en cuenta la frecuencia de utilización, la motivación, el uso concreto para tareas de investigación y el éxito en la obtención de información. Se buscó también conocer la visión del alumno sobre el papel del libro frente a la disponibilidad de textos electrónicos.

En cuanto a los aspectos del contexto sociocultural del alumno y su contribución a la adquisición de fluidez en tecnologías de información y comunicación, se intentó identificar los recursos disponibles en el entorno doméstico relacionados con dichas tecnologías, así como las características de la iniciación a las mismas, tales como la edad en que se aprende a usar el ordenador y la asistencia a clases extraescolares de informática. Otros aspectos del ámbito extraescolar incluyen la fluidez en inglés, la experiencia laboral y las perspectivas profesionales de los alumnos. 


\section{Análisis de datos}

$\mathrm{El}$ análisis de datos se organiza en dos fases: en la primera se realiza una descripción de las opiniones aportadas por los estudiantes sobre las TIC, se intenta captar algunas dimensiones de su fluidez en estas tecnologías y se describe el uso que hacen de Internet y de la biblioteca a la hora de buscar información para la investigación, procurando cotejar estos aspectos.

En la segunda fase del análisis se pretende hallar indicios del impacto generado por el origen social, el sexo y las características del contexto cultural del estudiante en la adquisición de habilidades en TIC, así como la actitud de los alumnos frente a dichas tecnologías. En lo que se refiere al entorno cultural se tiene en cuenta la importancia del papel del aprendizaje temprano de las TIC, la asistencia a clases extraescolares de informática y la fluidez en inglés.

\subsection{Las TIC y la biblioteca en la búsqueda de información}

Inicialmente, abriendo la primera fase del análisis, se presentan los datos sobre las opiniones de los estudiantes respecto a su aprendizaje de tecnologías de información y comunicación. El 99,7\% de ellos están de acuerdo en la importancia que tienen las TIC en su carrera y están conformes con el hecho de haber adquirido en la facultad conocimientos sobre las mencionadas tecnologías, que consideran útiles para su profesión $(77,8 \%)$. Por otra parte, los alumnos también coinciden mayoritariamente en la idea de que precisarán de muchos más conocimientos de esta materia de los que ya adquirieron $(96,5 \%)$ y no se consideran bien preparados en TIC: el 61,8\% de ellos no se incluyen en el grupo de los alumnos más preparados en tecnología de información y comunicación.

A continuación se describen los resultados obtenidos en el estudio en cuanto a las opiniones de los estudiantes sobre sus conocimientos del software considerado básico para un alumno de graduación de cualquier área, de acuerdo con lo anteriormente mencionado.

Se observan cifras altas en lo que se refiere a conocimiento bueno y muy bueno de procesadores de textos (Word u otro: 88,6\%), hojas de cálculo (Excel u otra: $75,5 \%$ ) y correo electrónico (Outlook u otro: $75,8 \%$ ).

Aunque el porcentaje de estudiantes que se considera competente en software de navegación en la Web (Netscape u otro: 64,4\%) no es bajo, se observa que el número de alumnos que aún no dominan ese tipo de software es importante $(35,4 \%)$, y preocupante, dada la importancia de Internet en la actualidad.

Menos del 50\% de los alumnos creen que están bien preparados para utilizar el software de presentación visual (PowerPoint u otro), y menos de 30\% se consideran competentes en bases de datos (Access u otro).

Scire. $13: 2$ (jul.-dic. 2007) 139-157. ISSN 1135-3716. 
Los datos obtenidos respecto a la utilización de Internet, excluyendo el correo electrónico, indican una alta frecuencia, aunque el uso cotidiano aún no está generalizado: solamente $48,6 \%$ la emplean diariamente. A ese respecto hay que tener en cuenta los rápidos cambios que se producen en cuanto al uso de este recurso y recordar el periodo de recogida de datos: el primer semestre de 2004.

Con el fin de comprobar el uso que el estudiante hace de Internet para sus investigaciones en la facultad se plantearon diversas preguntas cuyo objetivo es medir la frecuencia y la facilidad de uso de diferentes recursos de la Web, así como el grado de satisfacción obtenida tras la búsqueda. Los resultados se muestran en la tabla I.

\begin{tabular}{|c|c|c|c|c|c|}
\hline Uso de Internet & \multicolumn{4}{|c|}{ Opciones } & $\begin{array}{c}\text { Total } \\
(\%)\left(n .^{\circ}\right)\end{array}$ \\
\hline & Nunca & $\begin{array}{c}\text { Algunas } \\
\text { veces }\end{array}$ & $\begin{array}{l}\text { Muchas } \\
\text { veces }\end{array}$ & Siempre & \\
\hline ¿Utiliza Internet para trabajos de la facultad? & 1,6 & 30,7 & 32,9 & 34,8 & $100(316)$ \\
\hline $\begin{array}{l}\text { ¿Es fácil encontrar informaciones } \\
\text { sobre un tema en Internet? }\end{array}$ & 0,9 & 17,7 & 44,4 & 37,0 & $100(316)$ \\
\hline $\begin{array}{l}\text { Cuando investiga en Internet, } \\
\text { ¿confía en los resultados? }\end{array}$ & 0,6 & 16,8 & 48,4 & 34,2 & $100(316)$ \\
\hline $\begin{array}{l}\text { ¿Está satisfecho con el resultado } \\
\text { de las búsquedas? }\end{array}$ & 0,6 & 27,2 & 49,7 & 22,5 & $100(316)$ \\
\hline ¿Utiliza buscadores como Google y/o Altavista? & 13,7 & 11,8 & 22,7 & 51,8 & 100 (313) \\
\hline $\begin{array}{l}\text { ¿Busca textos escritos en otras lenguas } \\
\text { además de la portuguesa? }\end{array}$ & 63,0 & 23,1 & 7,6 & 6,3 & $100(316)$ \\
\hline
\end{tabular}

Tabla I. Uso de Internet para investigaciones de la facultad.

Los datos expuestos en la tabla I sugieren que los estudiantes utilizan bastante Internet para tareas de investigación de la facultad, no parecen tener dificultades para encontrar la información que precisan, tienen mucha confianza en su capacidad para buscar información, así como en los resultados de sus búsquedas, y utilizan mucho los buscadores, aunque sus investigaciones estén muy restringidas al idioma portugués. Cabe aclarar que, si bien la gran limitación de las investigaciones a la lengua portuguesa es preocupante, los resultados generales respecto al uso de Internet sorprenden positivamente, ya que indican un cierto grado de familiaridad con este vehículo de comunicación y recuperación de información.

En la tabla II se indica la opinión de los alumnos sobre el uso de Internet como sustituto de la biblioteca para sus investigaciones.

Los datos de la tabla II demuestran que la gran mayoría de los estudiantes consideran que, a pesar de la existencia de Internet, es necesario investigar en los libros para realizar los trabajos de la facultad. 


\begin{tabular}{|l|c|}
\hline Opinión & $\%$ \\
\hline Estoy en total desacuerdo & 36,3 \\
\hline Estoy más bien en desacuerdo & 40,6 \\
\hline Estoy más bien de acuerdo & 15,8 \\
\hline Estoy totalmente de acuerdo & 7,3 \\
\hline Total $(\%)\left(n \cdot{ }^{\circ}\right)$ & $100(316)$ \\
\hline
\end{tabular}

No respondieron $(\mathrm{NR})=4$

Tabla II. Opinión: con la existencia de Internet ya no es necesario investigar en los libros para los trabajos de la facultad.

Casi el $90 \%$ de los estudiantes contestan afirmativamente a la pregunta $\ll_{i} \mathrm{Va}$ usted a la biblioteca de la Universidad?». Los motivos por los cuales frecuentan la biblioteca son variados, según se observa en la tabla III.

\begin{tabular}{|l|c|}
\hline Motivo principal & $\%$ \\
\hline Leer periódicos y revistas & 6,3 \\
\hline Estudiar para el examen con material propio & 29,2 \\
\hline Estudiar para el examen con material de la biblioteca & 18,8 \\
\hline Buscar material de la facultad para investigar & 42,2 \\
\hline Otro & 3,5 \\
\hline Total $(\%)\left(n .{ }^{\circ}\right)$ & $100(256)$ \\
\hline
\end{tabular}

$\mathrm{NR}=4 ; \mathrm{NA}$ (no acude) $=60$

Tabla III. Principal motivo por el que frecuenta la biblioteca.

Se observa en la tabla III que la opción más frecuente es la búsqueda de material, sea para investigar, sea para acceder a los libros indicados para estudiar las materias de la facultad. También es bastante habitual el uso de la biblioteca como lugar de estudio, sin la utilización de su material bibliográfico.

\begin{tabular}{|l|c|c|c|c|}
\hline Asistencia a la biblioteca & \multicolumn{3}{c|}{ Recurso a Internet } & $\begin{array}{c}\text { Total } \\
\text { (\%) (n. }{ }^{\circ}\end{array}$ \\
\hline & $\begin{array}{c}\text { Nunca o } \\
\text { algunas veces }\end{array}$ & $\begin{array}{c}\text { Muchas } \\
\text { veces }\end{array}$ & Siempre & \\
Raramente o algunas veces en el semestre & 47,3 & 30,5 & 22,7 & $33,2(93)$ \\
\hline Muchas veces al bimestre & 28,6 & 38,0 & 43,3 & $36,8(103)$ \\
\hline Muchas veces al mes o diariamente & 24,1 & 31,5 & 34,1 & $30,0(84)$ \\
\hline Total $(\%)\left(n .^{\circ}\right)$ & $100(91)$ & $100(92)$ & $100(97)$ & $100(280)$ \\
\hline
\end{tabular}

Tabla IV. Frecuencia de uso de la biblioteca frente a la utilización de Internet para la investigación.

Scire. $13: 2$ (jul.-dic. 2007) 139-157. ISSN 1135-3716. 
La tabla IV examina la relación entre la frecuencia con que se usa Internet a la hora de investigar y aquella con la que se recurre a la biblioteca para la misma finalidad.

Los resultados mostrados en la tabla IV indican cierta asociación entre las dos variables, ya que aquellos que usan muy poco Internet suelen estar también entre los que utilizan muy poco la biblioteca; por otra parte, los que hacen mayor uso de la biblioteca también recurren más a Internet. De esta manera, se puede concluir que los dos tipos de recursos para la búsqueda de información no compiten entre sí, sino que, por lo contrario, el uso de uno parece contribuir al del otro.

Esta mutua contribución se muestra de manera más evidente en la tabla V, donde se puede observar que los alumnos que tienen facilidad para encontrar información en Internet suelen estar entre los que se desenvuelven mejor a la hora de localizar datos en la biblioteca.

\begin{tabular}{|l|c|c|c|c|}
\hline Asistencia a la biblioteca & \multicolumn{3}{c|}{ Recurso a Internet } & Total \\
\hline & $\begin{array}{c}\text { Nunca o } \\
\text { algunas veces }\end{array}$ & $\begin{array}{c}\text { Muchas } \\
\text { veces }\end{array}$ & Siempre & \\
Raramente & 20,5 & 17,3 & 10,0 & $18,6(51)$ \\
\hline Algunas veces & 45,5 & 41,8 & 30,0 & $43,1(118)$ \\
\hline Muchas veces o siempre & 34,0 & 40,8 & 60,0 & $38,3(105)$ \\
\hline Total $(\%)\left(n .{ }^{\circ}\right)$ & $100(156)$ & $100(98)$ & $100(100)$ & $100(274)$ \\
\hline
\end{tabular}

Tabla V. Facilidad para encontrar información en la biblioteca frente a la de localizar datos en Internet para la investigación.

A modo de síntesis se puede decir que esta primera etapa del análisis de datos sugiere que los alumnos estudiados, de un modo general, conceden gran importancia a las TIC, que tienen acceso a algunos de los recursos de las tecnologías de información y comunicación considerados como básicos y que muestran un cierto grado de familiaridad con Internet incluso para llevar a cabo sus investigaciones. Por otra parte, valoran la biblioteca como una fuente de información y no existen pruebas de esta que se haya sustituido por Internet en la investigación.

\subsection{Búsqueda de relaciones entre las variables origen social, sexo y contexto cultural de los estudiantes con respecto al uso de las TIC}

Esta segunda parte del análisis busca indicios de la influencia de los factores socioculturales y el sexo de los alumnos en su grado de fluidez en TIC, teniendo en cuenta concretamente la familiaridad y el uso de Internet para el trabajo de investigación. 
Inicialmente se considera la influencia del origen social del alumno, que se observa a partir del nivel educativo de la madre. A este respecto es importante mencionar que una proporción considerable de la población estudiada procede de familias cuyas madres no han terminado la primaria (35\%), y solo el $14 \%$ aproximadamente de las madres de esos alumnos tienen nivel universitario. De este modo, se hace evidente que existe una mayor concentración de alumnos de origen social bajo. No obstante, se observa una importante frecuencia de respuestas de estudiantes cuyas madres poseen diferentes grados de enseñanza, lo que permite observar la relación entre el nivel educativo de la madre y ciertos aspectos del uso y la familiaridad con Internet, tal como se muestra en la tabla VI (1).

\begin{tabular}{|l|c|c|c|c|c|c|}
\hline Uso de Internet & \multicolumn{5}{|c|}{ Nivel educativo de la madre } & Total \\
\hline & $\begin{array}{c}\text { Primaria }\left(\mathbf{n} .^{\circ}\right) \\
\text { incompleta } \\
\text { Siempre usa Internet }\end{array}$ & $\begin{array}{c}\text { Primaria } \\
\text { completa } \\
41,1\end{array}$ & $\begin{array}{c}\text { Secundaria } \\
\text { completa } \\
44,1\end{array}$ & $\begin{array}{c}\text { Superior } \\
\text { completa } \\
57,8\end{array}$ & 58,7 & $48,2(149)$ \\
\hline $\begin{array}{l}\text { Siempre usa Internet en las investigaciones } \\
\text { de la facultad }\end{array}$ & 33,0 & 34,5 & 36,7 & 37,0 & $35,0(107)$ \\
\hline $\begin{array}{l}\text { Siempre encuentra fácilmente información } \\
\text { en Internet }\end{array}$ & 37,6 & 31,1 & 35,6 & 52,2 & $37,9(116)$ \\
\hline $\begin{array}{l}\text { Siempre investiga en Internet y confía } \\
\text { en los resultados }\end{array}$ & 29,4 & 29,5 & 40,0 & 45,7 & $35,0(107)$ \\
\hline Siempre utiliza buscadores & 46,7 & 50,0 & 47,8 & 71,7 & $51,5(156)$ \\
\hline $\begin{array}{l}\text { Busca textos escritos en idiomas distintos } \\
\text { del portugués }\end{array}$ & 55,6 & 18,0 & 45,5 & 56,6 & $36,2(117)$ \\
\hline
\end{tabular}

Tabla VI. Uso de Internet destinado a la investigación según el nivel educativo de la madre.

El análisis de la tabla VI sugiere que existe cierta relación entre el origen social del alumno y los aspectos de habilidad en TIC estudiados. Esta variable parece afectar de algún modo al uso frecuente de Internet, pero no a su empleo para la investigación en la facultad, que es similar para los alumnos de diferentes orígenes sociales. El efecto del origen social parece más evidente con respecto a la confianza en el éxito de la investigación y en el uso de buscadores. La investigación en lengua extranjera presenta un patrón diferente: es más intensa en los dos extremos, esto es, entre los alumnos de origen social más alto y entre los del más bajo.

La siguiente fase del análisis tiene por objeto identificar la relación existente entre la fluidez en TIC y el sexo de los alumnos. En el grupo estudiado hay aproximadamente un $60 \%$ de mujeres y un $40 \%$ de hombres, un porcentaje de mujeres un poco mayor que el obtenido en otras investigaciones sobre la titulación en Scire. 13 : 2 (jul.-dic. 2007) 139-157. ISSN 1135-3716. 
Administración. De todas maneras, se trata de un área que se aproxima al equilibrio de género.

La tabla VII muestra la familiaridad con Internet y su uso destinado a la investigación según el sexo del estudiante.

\begin{tabular}{|l|c|c|c|}
\hline Uso de Internet & \multicolumn{2}{|c|}{ Sexo } & Total \\
\hline & Masculino (n. ${ }^{\circ}$ & Femenino & \\
Siempre usa Internet & 52,0 & 46,4 & $67,7(214)$ \\
\hline Siempre usa Internet en las investigaciones de la facultad & 27,2 & 39,8 & $34,8(110)$ \\
\hline Siempre encuentra fácilmente información en Internet & 40,0 & 35,1 & $37,0(117)$ \\
\hline Siempre investiga en Internet y confía en los resultados & 36,8 & 32,5 & $48,9(154)$ \\
\hline Siempre utiliza buscadores & 48,8 & 53,7 & $51,8(162)$ \\
\hline Busca textos escritos en idiomas distintos del portugués & 47,2 & 30,4 & $37,0(117)$ \\
\hline
\end{tabular}

Tabla VII. Uso de Internet destinado a la investigación según el sexo.

El examen de la relación entre la familiaridad con Internet y el sexo de los alumnos muestra situaciones contradictorias. Los varones utilizan Internet con más frecuencia; sin embargo, las mujeres la usan más para la investigación. Por otra parte, aquellos muestran algo más de facilidad para encontrar información, utilizar buscadores y extraer datos de textos escritos en lengua extranjera. Parece existir una tendencia al uso más autónomo y diversificado de Internet por parte de los varones. Esos indicios de las diferencias de género en relación con el uso de TIC son reforzados por otros datos obtenidos en la investigación que sugieren mejores perspectivas respecto al uso de TIC entre los hombres. Estos tienden a tener mayor acceso a tipos de software distintos de los convencionales y a instalarlos con más frecuencia que las mujeres. Los varones también tienden a considerarse más competentes en TIC y a creer que tienen mejores perspectivas profesionales. Este fenómeno se observa incluso a pesar de que ambos sexos se desenvuelven de un modo similar y de que las mujeres desean continuar sus estudios y realizar cursos de nivel superior, como la maestría y el doctorado, en mayor proporción que los hombres: el 84,1\% de las mujeres tienen intención de seguir estudiando, frente al $81,6 \%$ de los varones. Los datos obtenidos en el presente estudio también indican un grado de inserción en el mercado laboral alto y equivalente entre ambos sexos: el $94,4 \%$ de los hombres y el $91,1 \%$ de las mujeres trabajan.

En esta última fase del análisis se intenta captar la relación existente entre algunos aspectos que pueden ser considerados como aquellos que contribuyen a la formación del llamado capital cultural, el cual también favorece el acceso a las TIC y la ampliación de las perspectivas profesionales de los alumnos. Según se ha in- 
dicado anteriormente, para ello se tuvieron en cuenta algunos aspectos de la experiencia preuniversitaria con tecnologías de información y comunicación, los cuales reflejan la inversión específica del alumno y de las familias en esta área. Para ello se consideró el acceso temprano al ordenador en el ámbito doméstico y la asistencia a clases de informática, así como la fluidez en lengua inglesa, un recurso cultural que, si bien no está directamente ligado a las TIC, puede contribuir al conocimiento de dichas tecnologías, y que también exige una inversión por parte del alumno y de su familia en clases extraescolares.

En relación con la edad en la que los alumnos comenzaron a utilizar el ordenador, se observa una variación considerable entre ellos: un gran porcentaje de alumnos tuvieron acceso a su uso antes de los 14 años (24,9\%), mientras que otros solamente lo tuvieron a partir de los $18(38,7 \%)$. Esos resultados parecen reflejar no solo la diferencia entre las condiciones sociales y las características culturales de las familias, sino también el hecho de que la presencia de un ordenador en los ámbitos escolar y doméstico es un fenómeno reciente y en clara expansión.

La asistencia a clases de informática se revela como una estrategia generalizada, un patrón común entre los alumnos estudiados, ya que un gran porcentaje de ellos la utilizaron. En cuanto a la fluidez en inglés, se nota una menor preparación, pues pocos se consideran competentes en este sentido.

Dos de los factores arriba mencionados - la edad de iniciación en el uso del ordenador y la asistencia a clases de informática — no tienen el efecto esperado respecto a la familiaridad con Internet. Los estudiantes que se iniciaron temprano en el uso del ordenador y los que no lo hicieron se cuentan en una proporción similar entre los que siempre utilizan Internet, entre los que la utilizan para buscar información y incluso entre los que hacen uso de buscadores. Sorprendentemente, la asistencia a clases de informática tampoco tiene el efecto previsto en cuanto a la familiaridad con Internet. De hecho, un porcentaje mayor de alumnos que no

\begin{tabular}{|l|c|c|c|c|c|}
\hline Uso de Internet & \multicolumn{3}{|c|}{ Fluidez de lectura en inglés } & Total \\
(\%) (n. ${ }^{\circ}$ )
\end{tabular}

Tabla VIII. Uso de Internet destinado a la investigación según la fluidez de lectura en inglés.

Scire. $13: 2$ (jul.-dic. 2007) 139-157. ISSN 1135-3716. 
asistieron a estas clases muestran más familiaridad con los aspectos del uso de Internet que estamos estudiando. Sin embargo, de los factores considerados, la fluidez en lengua inglesa es el que está más fuertemente vinculado a la familiaridad con Internet. Llama la atención el alto porcentaje de uso frecuente de Internet entre quienes se consideran competentes en inglés. Lo mismo sucede entre aquellos que tienen facilidad para encontrar información y entre los que utilizan buscadores, como muestra la tabla VIII.

\section{Consideraciones finales}

Los alumnos de Administración, que han sido el objeto de este estudio, se muestran conscientes de la importancia de las TIC en su vida profesional y revelan haber tenido ya acceso a estas tecnologías en sus tareas de investigación: conocen razonablemente el software considerado básico para cualquier alumno de graduación y tienen la costumbre de utilizar Internet en sus investigaciones. Un porcentaje considerable de los estudiantes dice conocer y utilizar diversos recursos disponibles en Internet, como los motores de búsqueda o buscadores. Sin embargo, pocos se consideran capacitados para utilizar documentos escritos en lengua inglesa en sus investigaciones.

A pesar de que el motivo de uso de la biblioteca mencionado con mayor frecuencia es la investigación, es relativamente alto el número de alumnos que acuden a la ella como lugar de estudio y con su propio material. Se intentó averiguar si el aumento del uso de Internet en la investigación tendría un efecto negativo en la utilización de la biblioteca, pero no parece ser así. Se muestra cierta tendencia a una relación de sentido positivo entre ambas variables, ya que los alumnos que más recurren a Internet son también los que más emplean la biblioteca. Del mismo modo, aquellos que tienen más facilidad para encontrar información en Internet tienden también a desenvolverse mejor en la biblioteca.

Las características arriba descritas en cuanto al acceso a las TIC se refieren, por lo general, a alumnos de origen social relativamente poco favorecido, pues la mayor parte de las madres de estos estudiantes no tienen nivel universitario, y un porcentaje relativamente grande de ellas ni siquiera terminaron la primaria.

A la hora de identificar los factores que pueden contribuir a la fluidez en TIC, destacaron los efectos de la educación de la madre, del sexo del alumno y del acceso a determinados recursos extrauniversitarios, como el acceso temprano al ordenador, la asistencia a clases de informática y la competencia en inglés. Mediante el análisis de estos factores se obtuvieron algunos resultados que merecen ser mencionados.

Se observó cierto grado de relación entre la educación de la madre y la fluidez en tecnologías. Se verificaron también diferencias de comportamiento en cuan- 
to al uso de Internet dependiendo del sexo de los alumnos: los varones la utilizan más frecuentemente, su uso les resulta más fácil y confían un poco más en los resultados, lo que sugiere mayor autonomía y diversidad en el uso de la Red entre ellos que entre las mujeres. Estas suelen enfocar el uso de Internet a las tareas de investigación.

Entre los factores que pueden indicar la presencia de un capital cultural capaz de favorecer el uso de Internet destaca la fluidez en inglés. Este aspecto resultó tener mayor relación con la familiaridad con el uso de Internet que otros, como por ejemplo la asistencia a clases de informática.

Los resultados refuerzan los argumentos presentados en el inicio del trabajo respecto a la diversidad de factores culturales que pueden estar relacionados con la adquisición de fluidez en tecnologías de información y comunicación.

\section{Notas}

(1) En las tablas VI y VII los datos representan la síntesis del cruce de diversas variables. Así, para facilitar la lectura, en cada línea se indican solamente las respuestas correspondientes a los alumnos que están muy familiarizados con el uso de Internet. La comparación de los resultados debe realizarse mediante una lectura horizontal. Así, por ejemplo, en la tabla VI se lee que, de los alumnos que usan siempre Internet, el 41,1\% tienen madres que no han terminado la primaria, el 54,1\% tienen madres que sí la han completado, el 47,8\% tienen madres que han acabado la secundaria y el 58,7\% tienen madres con titulación superior. La lectura debe realizarse de este modo para todos los aspectos estudiados en cuanto a la familiaridad con Internet. Si hay relación entre el nivel educativo de la madre y el uso de Internet, los porcentajes deben aumentar a medida que aumenta el grado de educación de la madre.

\section{Referencias}

Bonder, Gloria (2001). The new information technologies and women: essential reflections. Santiago de Chile: CEPAL, Naciones Unidas (serie "Mujer y Desarrollo", 39), 2001.

Bourdieu, P. (1979). La distinction: critique sociale du jugement. París: Minuit, 1979.

Bourdieu, P. (1987). What makes a social class? On the theoretical and practical existence of groups. // Berkeley Journal of Sociology. 32 (1987) 1-49.

Castells, Manuel (2003). A galáxia da Internet: reflexões sobre a Internet, os negócios e a sociedade. Río de Janeiro: Jorge Zahar, 2003.

Chen, D. H. C. (2004). Gender equality and economic development. World Bank policy research working paper. Abril de 2004.

Chu, Clara M. (2002). The digital divide: A resource list. http://www.gseis.ucla.edu/ faculty/chu/digdiv/ (2002-12-22).

Committee on Information Technology Literacy (1999). CSTB (National Academy of Sciences). Being fluent with information technology. Washington, DC: National Academy Press, 1999. http://www.nap.edu/books/03090 6399X/html/ (2003-01-21).

Scire. $13: 2$ (jul.-dic. 2007) 139-157. ISSN 1135-3716. 
Daniel, Graene; Cox, Kelvin (s. f.). Computer literacy for workplace. http://wwwtools.cityu. edu.hk/newslett/Computerliteracyworkplace.htm (2002-12-04).

Diniz, Cládice N. (2005). A fluência em tecnologia da informação entre estudantes de Administração. Tese de doutorado em Ciências da Informação, aprovada pela Escola de Comunicação da Universidade Federal do Rio de Janeiro em março de 2005. Orientadora: Gilda Olinto. Río de Janeiro: UFRJ, Convênio UFRJ/ECO-MCT/IBICT, 2005.

ECLAC (Economic Commission for Latin America and the Caribean) (2003). Road maps towards an information society in Latin America and the Caribbean. Santiago de Chile, 2003.

García, J.; Escamilla, R. R.; Reyes, G. A. (2004). Aunque la mona tenga posgrados, mona se queda: la falta de equidad de género en el mercado laboral. // V Congreso Iberoamericano de Ciencia, Tecnología y Género. México: UNAM, 2004.

Hatschbach, Maria Helena de L. (2002). Information literacy: aspectos conceituais e iniciativas em ambiente digital para o estudante de nível superior. Dissertação de Mestrado em Ciências da Informação, aprovada pela Escola de Comunicação da Universidade Federal do Rio de Janeiro, em novembro de 2002. Orientadora: Gilda Olinto. Río de Janeiro: UFRJ, Convênio UFRJ/ECO-MCT/IBICT, 2002.

Inep/MEC (Instituto Nacional de Estudos e Pesquisas Educacionais do Ministério da Educação) (2002). Censo de Educação Superior 2002. Brasilia: Inep/MEC, 2002. Base de datos. http://www.inep.gov.br/superior/censosuperior/ (2004-09-24).

Mann, C. L. (2003). Information technologies and international development: conceptual clarity in the search for commonality and diversity. // Information Technologies and International Development. 1:2 (2003) 67-79.

Martínez, J. (2005). El empleo en los servicios financieros. Costa Rica: buenas y no tan buenas noticias. Santiago de Chile: CEPAL, Naciones Unidas (serie "Mujer y Desarrollo", 62), 2005.

Mello, H. P.; Lastres, H. M. M.; Marques, T. C. N. (2003). As mulheres brasileiras no sistema de ciência, tecnologia e inovação. Mimeo, 2003.

OCDE (Organização para a Cooperação e Desenvolvimento Econômico) (2002). Perspectivas da tecnologia da Informação na OCDE. Mimeo, 2002.

Olinto do Valle Silva, Gilda (1994). Reprodução de classe e produção de gênero através da cultura. Tese de doutorado orientada por Aldo de Albuquerque Barreto, apresentada ao Programa de Pós-Graduação em Comunicação da Universidade Federal do Rio de Janeiro - ECO/UFRJ. Río de Janeiro, 1994.

Olinto, Gilda (2003). Mulheres e jovens na liderança da pesquisa. // V Encontro Nacional da Pesquisa em Ciência da Informação (Belo Horizonte, 2003). Anais do V ENANCIB, 2003. CD-ROM.

Olinto, Gilda (2004). La inserción de las mujeres em la investigación científica y tecnológica en Brasil: indicios de transformación. // V Congreso Iberoamericano de Ciencia, Tecnología y Género. México: UNAM, 2004.

Pohjola, M. (2002). New economy in growth and development. World Bank, WIDER, working paper 67, 2002.

Scire. 13 : 2 (jul.-dic. 2007) 139-157. ISSN 1135-3716. 
Quiñones, M. (2005). Demandas de capacitación del sector financiero. Sesgos de género y evaluación por competencias. Santiago de Chile: CEPAL, Naciones Unidas (serie "Mujer y Desarrollo", 61), 2005.

Sen, Amaya (1999). Desenvolvimento como liberdade. São Paulo: Cia das Letras, 1999. 